\title{
Estudo SAIMI - Saúde e Acesso aos Serviços de Saúde dos Imigrantes do Subcontinente Indiano em Lisboa: Que Recomendações para Cuidados de Saúde Equitativos e Culturalmente Adaptados?
}

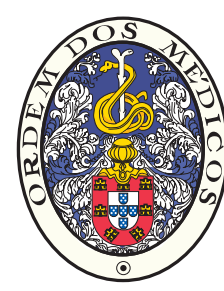

\author{
SAIMI Study - Health and Health Care Access by Immigrants from the Indian \\ Subcontinent in Lisbon: What Recommendations for Equitable and Culturally \\ Adequate Health Care?
}

\author{
Inês CAMPOS MATOS ${ }^{1}$, Violeta ALARCÃO ${ }^{1}$, Elisa LOPES ${ }^{1}$, Carla OIKO ${ }^{1}$, Mário CARREIRA ${ }^{1}$ \\ Acta Med Port 2015 Mar-Apr;28(2):164-176
}

\section{RESUMO}

Introdução: O crescimento da população imigrante em Portugal tem sido consistente nas últimas décadas. Apesar disto, a informação sobre a saúde das populações imigrantes é escassa. Esta investigação utiliza dados recolhidos junto da população oriunda do subcontinente indiano a residir no distrito de Lisboa para produzir recomendações para a prestação de serviços de saúde culturalmente adaptados.

Material e Métodos: Estudo transversal junto da comunidade imigrante do subcontinente indiano (Bangladesh, Índia e Paquistão) a residir em Lisboa, selecionada com base numa técnica de amostragem bola de neve e recorrendo a inquiridores com acesso privilegiado à população-alvo. O questionário inquiriu sobre a saúde, o acesso aos cuidados de saúde, estilos de vida e atitudes perante a morte. Foi feita uma análise descritiva dos dados e uma comparação entre as três nacionalidades padronizada para a idade.

Resultados: Foram administrados questionários a 1011 indivíduos com uma taxa de adesão de 97\%. A maioria dos participantes eram adultos do sexo masculino. Os imigrantes indianos relataram mais frequentemente barreiras na utilização dos serviços de saúde e tinham uma maior frequência de doenças crónicas. Os imigrantes paquistaneses tinham piores indicadores de estilos de vida.

Discussão: A população imigrante do subcontinente indiano tende a relatar mais dificuldades linguísticas no acesso aos cuidados de saúde quando comparada com outras populações imigrantes. Com base em recomendações da Organização Mundial da Saúde, foi possível adaptar este conhecimento para produzir recomendações adaptadas ao contexto português.

Conclusão: Existem diversos aspetos na gestão dos serviços de saúde em Portugal que podem ser melhor adaptados à população imigrante do subcontinente indiano.

Palavras-chave: Emigrantes e Imigrantes; Oeste Asiático; Portugal; Recomendações para o Planeamento em Saúde.

\section{ABSTRACT}

Introduction: The growth of the immigrant population in Portugal has been consistent over the past decades. Nevertheless, information on the health of immigrant populations is scarce. This research uses data collected from the population from the Indian subcontinent living in the district of Lisbon to produce recommendations for the provision of culturally adapted health services.

Material and Methods: Cross-sectional study with the immigrant community of the Indian subcontinent (Bangladesh, India and Pakistan) living in Lisbon, selected based on a snowball sampling technique and using privileged access interviewers. The questionnaire focused on health, health care access, lifestyle and attitudes towards death. The data were subject to a descriptive analysis and an age-standardized comparison between the three nationalities was made.

Results: Surveys were administered to 1011 individuals with a participation rate of $97 \%$. Most participants were adult males. Indian immigrants most frequently reported barriers to use of health services and had a higher frequency of chronic diseases. Pakistani immigrants had worse lifestyle indicators.

Discussion: The immigrant population from the Indian subcontinent tends to report more language difficulties in health care access when compared with other immigrant populations. Based on recommendations of the World Health Organization, it was possible to adapt this knowledge to produce recommendations adapted to the Portuguese context.

Conclusion: There are several aspects in the management of health services in Portugal that can be better adapted to the immigrant population from the Indian subcontinent.

Keywords: Asia, Western; Emigrants and Immigrants; Health Planning Guidelines; Portugal.

\section{INTRODUÇÃO}

Nas últimas décadas, Portugal consolidou-se como um País de destino para pessoas de diversas origens - entre 2001 e 2011 o número de estrangeiros em Portugal duplicou. ${ }^{1}$ Apesar desta tendência migratória, existe uma escassez de informação sobre as atitudes e comportamentos dos diferentes grupos de imigrantes em relação à saúde e à doença e sobre os determinantes do seu acesso aos serviços de saúde. Esta ausência de dados científicos é particularmente importante dada a vulnerabilidade destas populações para as questões de saúde, que enfrentam

1. Unidade de Epidemiologia. Instituto de Medicina Preventiva e Saúde Pública. Faculdade de Medicina. Universidade de Lisboa. Lisboa. Portugal.

Recebido: 12 de Junho de 2014 - Aceite: 18 de Agosto de 2014 | Copyright @ Ordem dos Médicos 2015 
frequentemente riscos acrescidos e barreiras no acesso aos cuidados de saúde.

Segundo os dados de 2012 do Serviço de Estrangeiros e Fronteiras, dois em cada 100 residentes regulares em Portugal com nacionalidade estrangeira eram oriundos do Bangladesh (1351), Índia (5657) e Paquistão (2425), a maior parte dos quais no distrito de Lisboa. ${ }^{2}$ Este número é, provavelmente, subestimado já que estes dados não contemplam os imigrantes em situação irregular nem os naturalizados. Apesar de não constituírem um grupo muito expressivo, o número de imigrantes do subcontinente indiano tem vindo a aumentar e tem características particulares face às restantes populações imigrantes (predominantemente do género masculino, jovens e muçulmanos). ${ }^{3}$

Até muito recentemente o tema imigração e saúde foi negligenciado tanto na área da saúde como na área dos estudos migratórios, ${ }^{4,5}$ existindo apenas alguns trabaIhos desenvolvidos junto de comunidades imigrantes com amostras reduzidas e frequentemente não representativas.

Os dados existentes, no entanto, sugerem que a população imigrante em Portugal tem menor acessibilidade aos serviços de saúde quando comparada com a população não migrante. ${ }^{6}$ Vários fatores foram identificados estando associados a uma menor utilização dos cuidados de saúde: menos tempo de residência em Portugal, ${ }^{6-8}$ residência ilegal, ${ }^{7-9}$ país de origem, nomeadamente africano (quando comparado com leste europeu) ${ }^{8}$ e sexo masculino. ${ }^{9}$ Foram documentadas situações em que os indivíduos fornecem nomes e moradas falsas nos serviços de saúde com receio de serem identificados pelas autoridades. ${ }^{10}$ Adicionalmente, apesar de não permitido legalmente, foram documentadas situações em que centros de saúde recusaram o atendimento de imigrantes não documentados. ${ }^{10}$ Um estudo junto de profissionais de saúde portugueses mostrou ainda que existem oportunidades de intervenção junto destes no contexto de diversidade cultural, já que a maioria considerou ter reduzidas competências socioculturais para lidar com estas populações. ${ }^{11}$

As comunidades de imigrantes do subcontinente indiano são relativamente pouco estudadas. A maioria dos estudos existentes na área da saúde tem como cenário os EUA, onde estas comunidades têm uma grande representatividade, e incluem estudos e descrições sobre a importância de fatores culturais no acesso e utilização dos cuidados de saúde. ${ }^{12-14}$

Apesar de existirem diferenças culturais entre as várias comunidades de imigrantes oriundos do Bangladesh, Índia e Paquistão, existem também características comuns que podem influenciar a procura e utilização dos cuidados de saúde. Destas características, destaca-se o elevado suporte familiar (que pode implicar uma menor necessidade de serviços de saúde mental, mas também limitar a liberdade individual dos seus elementos), ${ }^{14}$ a tendência para uma importante utilização de medicinas tradicionais, principalmente por indivíduos com menor capacidade de falar a língua do país ${ }^{13}$ e a rejeição do transplante de órgãos. ${ }^{15}$ As recomendações para boas práticas clínicas são frequentemente muito genéricas, mas geralmente lembram a necessida- de de conhecer as origens culturais do doente, ${ }^{12}$ adaptar o ónus da decisão clínica adequadamente (do doente para a família, por exemplo) e acautelar para não agir com base em estereótipos. ${ }^{16}$

Em Portugal, desconhecemos a existência de estudos relacionados com a saúde destas comunidades. Existem alguns estudos com enfoque nas comunidades indianas sobre trajetórias sociais e estratégias empresariais, ${ }^{17,18} \mathrm{O}$ papel da religião ${ }^{19}$ e processos de identidade. ${ }^{20-23}$ Estes estudos são de carácter qualitativo e exploram estratégias de adaptação destas comunidades a um novo país.

Urge assim identificar as principais necessidades de saúde das comunidades imigrantes do subcontinente indiano, as principais condicionantes do acesso aos cuidados de saúde e determinar as limitações de resposta dos serviços públicos a estas especificidades. Esta reflexão ganha particular importância no atual contexto Português de crise financeira, já que nestes momentos existe uma tendência para reduzir os custos dos cuidados de saúde com populações minoritárias. ${ }^{24}$

O Comité Regional Europeu da Organização Mundial de Saúde (OMS - Europa) resumiu, em 2010, a evidência existente sobre boas práticas na adaptação dos cuidados de saúde às necessidades das populações imigrantes, reconhecendo simultaneamente a falta de informação para concluir com alguma certeza que intervenções são verdadeiramente eficazes. ${ }^{25} \mathrm{~A}$ Tabela 1 resume estas recomendações no que se referem à prestação de serviços, gestão de recursos e financiamento dos serviços de saúde.

Em Portugal, o II Plano para a Integração dos Imigrantes $2010-2013^{26}$, incluía uma lista de seis medidas relacionadas com a saúde das populações migrantes, incluindo:

(i) Promoção do acesso dos imigrantes ao Serviço Nacional de Saúde (SNS);

(ii) Plano de formação para a interculturalidade dos profissionais do SNS;

(iii) Implementação de um programa de serviços de saúde amigos dos imigrantes;

(iv) Desenvolvimento de parcerias para a promoção do acesso dos imigrantes à saúde em Portugal;

(v) Institucionalização de procedimentos com vista a uma melhor gestão dos acordos de saúde e agilização do acesso dos imigrantes e seus acompanhantes aos serviços de saúde;

(vi) Investimento da promoção da saúde mental dos imigrantes.

A avaliação da implementação deste plano é, no entanto, limitada pela falta de informação estatística e a sua dispersão por diferentes entidades. ${ }^{5}$

Partindo das recomendações da OMS - Europa, este estudo tem como objetivo integrar o conhecimento existente sobre o contexto português e dados obtidos junto da comunidade imigrante do subcontinente indiano para produzir recomendações direcionadas à prestação de serviços e gestão de recursos do SNS. Trata-se de uma reflexão que, espera-se, ajude a promover boas práticas direcionadas para a população imigrante em Portugal. 
Tabela 1 - Recomendações emitidas pela Comité Regional Europeu da Organização Mundial de Saúde (OMS - Europa) para as boas práticas dos sistemas de saúde na promoção da saúde dos imigrantes

Prestação de Serviços (incluindo promoção e educação para a saúde, cuidados preventivos e rastreios, curativos e paliativos)
- Monitorizar a acessibilidade e qualidade da prestação dos serviços de saúde para populações migrantes

- Identificar e analisar problemas, criar soluções e avaliar a sua eficácia

- Reduzir as barreiras linguísticas oferecendo serviços de interpretação e materiais traduzidos quando necessário

- Reduzir barreiras administrativas e práticas ao acesso

- Adaptar a prestação de serviços às diferenças culturais e sociais

- Afetar recursos de acordo com a incidência e seriedade dos problemas de saúde

- Utilizar uma abordagem centrada e orientada para fornecer informação sobre saúde à população imigrante

- Adotar uma abordagem que abranja todo o sistema para a eliminação de discriminação institucional e o desenvolvimento de "competências culturais"

- Aumentar a cooperação intersectorial com outros serviços

- Envolver as comunidades imigrantes no desenho, implementação, monitorização e avaliação dos serviços de saúde

- Fortalecer a prestação de cuidados primários de saúde e reduzir a dependência inapropriada dos serviços de emergência

- Integrar os serviços de saúde para imigrantes tanto quanto possível nos serviços para o resto da população de forma a facilitar o controlo, garantir a sustentabilidade e evitar a institucionalização da exclusão social

Geração de Recursos

- Desenvolver uma força de trabalho capaz pela formação e educação sobre migração, etnia e saúde; os programas não devem ser exclusivos para os profissionais de saúde mas incluir também investigadores, gestores, administradores e policy-makers

- Formalizar funções como "mediadores culturais", "intérpretes para a saúde" e "trabalhadores comunitários de saúde"

- Estabelecer sistemas para a geração e disseminação do conhecimento sobre a saúde da população imigrante

- Fortalecer e consolidar o conhecimento dos investigadores e profissionais de saúde promovendo a cooperação entre disciplinas, profissões e sectores de saúde
Financiamento
- Reduzir as barreiras financeiras dos cuidados de saúde para os mais desfavorecidos limitando os pagamentos diretos e promovendo a cobertura universal

- Assegurar que os imigrantes que recorrem ao serviço de saúde conhecem os seus direitos que estes são respeitados pelos prestadores de serviços

- Investigar os custos económicos das iniquidades em saúde e de diagnósticos inadequados, tratamento desadequado, erros médicos, abandono e má adesão terapêutica que resultem de um serviço cultural e linguisticamente desadequado.

\section{MATERIAL E MÉTODOS}

Estudo transversal com imigrantes do Bangladesh, Índia e Paquistão residentes no distrito Lisboa. Foram considerados elegíveis todos os imigrantes que deixaram o seu país de origem com a intenção de residir temporária ou permanentemente em Portugal (primeira geração), assim como os que nasceram em Portugal, filhos de pais imigrantes (segunda geração), mesmo que tenham adquirido a nacionalidade Portuguesa. Foram excluídos os indivíduos que, apesar de terem naturalidade do Bangladesh, Índia ou Paquistão, fossem filhos de Portugueses emigrados nesses países que entretanto tenham regressado a Portugal.

Foi utilizado um método de amostragem não probabilístico através da técnica bola de neve, que consiste em pedir a indivíduos elegíveis para o estudo que identifiquem e contactem ou facultem contactos de outros potenciais participantes. Estes novos indivíduos são contactados e são-lhes igualmente solicitados novos contactos até a obtenção da amostra pretendida. Para iniciar o processo de amostragem bola de neve partiu-se de contactos já estabe- lecidos com instituições de acesso privilegiado a estas populações. Para aumentar a heterogeneidade da amostra, os contactos iniciais incluíram representantes de todos os subgrupos importantes: naturalidade das três comunidades em estudo, estatuto de imigração diferenciado, diferentes tempos de estadia em Portugal, homens, mulheres, indivíduos de diferentes grupos etários e também de diferentes condições sociais e níveis de educação.

Foram administrados questionários individuais, recorrendo a inquiridores com acesso privilegiado à população-alvo (Privileged Access Interviewer Method), ou seja, inquiridores membros das comunidades em estudo. ${ }^{27}$

A administração dos questionários teve lugar entre novembro de 2012 e março de 2013, preferencialmente no domicílio ou num local que assegurasse a privacidade dos participantes. À exceção dos dados relativos aos menores de 15 anos, que foram obtidos através de um informador privilegiado (proxy), apenas foram considerados os dados recolhidos ao próprio.

O questionário teve por base uma seleção das questões 
utilizadas no $4^{\circ}$ Inquérito Nacional de Saúde (INS), tendo sido incluída na análise uma caracterização do acesso aos cuidados de saúde e da prestação efetiva de cuidados. $O$ questionário estava dividido em doze blocos que abrangiam os seguintes temas gerais: caracterização sociodemográfica, estado de saúde, estilos de vida, saúde oral, acesso aos cuidados de saúde, inventário de saúde mental e atitudes perante a morte.

Foi feita uma caracterização da amostra em estudo, utilizando métodos de estatística descritiva, em termos das suas características sociodemográficas, autoavaliação da qualidade de vida, estado de saúde e estilos de vida, saúde oral e hábitos de higiene oral, atitudes perante a morte e utilização dos serviços de saúde. Posteriormente fez-se uma análise dos dados sociodemográficos e de saúde ajustados para a idade, por comunidade.

O estudo foi aprovado pela Comissão de Ética da Faculdade de Medicina de Lisboa e pela Comissão Nacional de Proteção de Dados. Todos os participantes tiveram acesso a um consentimento informado, redigido em inglês ou português.

\section{RESULTADOS}

Foram administrados questionários a 1011 indivíduos, com uma taxa de adesão global de $97 \%$. A Tabela 2 resume as características sociodemográficas das populações de adultos e crianças do estudo. A maioria dos participantes eram adultos e do sexo masculino. Todos os adultos de nacionalidade Bangladeshiana e Paquistanesa eram naturais dos seus países de origem; 3,2\% (IC 95\% 0,9-5,5\%) dos adultos Indianos eram naturais de Portugal. Entre 61 e $64 \%$ dos indivíduos dos três grupos tinha uma profissão, sendo uma grande parte pertencente ao grupo profissional 'pessoal dos serviços e vendedores'. A mediana do tempo de residência em Portugal, em anos, para a população adulta foi de 1, 8 e 4 para o grupo de imigrantes Bangladeshianos, Indianos e Paquistaneses, respetivamente. Quase todos os Bangladeshianos e Paquistaneses declararam-se muçulmanos, enquanto os Indianos declararam ser hinduístas. Nas três comunidades, a maioria era 'muito pouco' ou 'muitíssimo' praticante da sua religião.

A Tabela 3 resume as respostas dos inquiridos de idade adulta no que diz respeito à autoavaliação da qualidade de vida, saúde mental, estilos de vida, estado de saúde e atitudes perante a morte.

Entre os Bangladeshianos, Indianos e Paquistaneses, 96,6\% (IC 95\% 94,4-98,8\%), 69,9\% (IC 95\% 63,9-75,8\%) e $95,2 \%$ (IC 95\% 92,3-97,9\%), respetivamente, classificaram a sua qualidade de vida como 'Boa' ou 'Muito boa'. Os adultos de nacionalidade indiana tinham maior frequência de doenças crónicas (diabetes, colesterol, hipertensão arterial e AVC), contrastando com os Paquistaneses, que apresentaram frequências mais baixas.

Apenas 9,6\% (IC 95\% 6,0-13,2\%), 15,8\% (IC 95\% 11,1$20,5 \%$ ) e $42,1 \%$ (IC 95\% 35,5-48,7\%) dos adultos Bangladeshianos, Paquistaneses e Indianos, respetivamente, autorizaria a doação de órgãos. A visão sobre a eutanásia e sobre a utilização prolongada de meios de suporte de vida também é variada entre nacionalidades, sendo que os adultos Bangladeshianos tenderam simultaneamente a ser menos recetivos à ideia de eutanásia [9,6\% (IC 95\% 6,0$13,2 \%)$ e da utilização prolongada de meios de suporte de vida [19,9\% (IC 95\% 15,1-24,7\%)] e os Indianos no outro extremo, mais recetivos a ambas as intervenções $[42,1 \%$ (IC 95\% 35,5-48,7\%) e 35,2\% (IC 95\% 28,9-41,6\%), respetivamente].

$\mathrm{Na}$ Tabela 4 estão descritos os dados sobre a saúde oral e utilização dos cuidados de saúde dos participantes (adultos e crianças) do estudo.

Poucos adultos referem nunca ter recorrido aos serviços de saúde [1,5\% (IC 95\% 0,03-2,9\%) Bangladeshianos, $3,2 \%$ (IC 95\% 0,9-5,5\%) Indianos e 0,4\% (IC 95\% 0,0-1,2\%) Paquistaneses], ou ter tido alguma dificuldade linguística de compreensão nos serviços de saúde [14,3\% (IC 95\% 10,1-18,5\%) Bangladeshianos, 26,9\% (IC 95\% 21,0-32,8\%) Indianos e 14,1\% (IC 95\% 9,6-18,6\%) Paquistaneses].

Trinta e seis adultos [5,0\% (IC 95\% 3,4-6,6\%)] referem ter-Ihes sido recusada a prestação de cuidados de saúde. Apenas seis inquiridos [0,8\% (IC 95\% 0,14-1,46\%)] referem ter sido tratados de forma discriminatória pelos profissionais de saúde por ser imigrante. A grande maioria da amostra de inquiridos adultos concordou com a afirmação que os profissionais de saúde, de uma maneira geral, compreendem bem as necessidades de saúde dos imigrantes [93,8\% (IC 95\% 92,0-95,6\%)].

A larga maioria dos participantes adultos [96,0\% (IC 95\% 94,6-97,5\%)] está inscrito num centro de saúde e, destes, 94,4\% (IC 95\% 92,7-96,1\%) tem médico de família. Cerca de $24,4 \%$ (IC 95\% 21,2-27,6\%) já tinham recorrido ao serviço de urgência.

Os adultos Indianos foram quem mais frequentemente referiu dificuldades económicas em comprar medicamentos, pagar consultas médicas ou meios complementares de diagnóstico nos últimos 12 meses [7,4\% (IC 95\% 3,9$10,9 \%$ ), 4,2\% (IC 95\% 1,5-6,9\%) e 3,7\% (IC 95\% 1,2-6,2\%), respetivamente]. Entre as crianças, apenas os Indianos referiram dificuldades económicas que impediram a compra de medicamentos [3,1\% (IC 95\% 0,1-6,1\%)] e consulta de médicos [1,5\% (IC 95\% 0,0-3,6\%)].

A Tabela 5 resume algumas características dos três grupos de diferentes nacionalidades, ajustadas para a idade. Os imigrantes indianos são quem está há mais tempo em Portugal e quem tem uma maior proporção de indivíduos nascidos em Portugal. É também este grupo quem mais identifica a existência de barreiras linguísticas à utilização dos cuidados de saúde que mais refere ter sido tratado de forma discriminatória por profissionais de saúde em Portugal. Os imigrantes do Bangladesh são quem tem uma maior proporção de indivíduos com o ensino superior completo mas também de desempregados. Apesar de avaliarem a sua qualidade de vida como "boa" ou "muito boa" com mais frequência, são quem mais frequentemente apresenta risco de sofrimento psicológico. Por fim, os imigrantes Paquistaneses destacam-se pela elevadíssima proporção de 


\begin{tabular}{|c|c|c|c|c|}
\hline & \multicolumn{4}{|c|}{$\begin{array}{l}\text { Imigrantes Bangladeshianos } \\
\qquad(n=346)\end{array}$} \\
\hline & \multicolumn{2}{|c|}{$\begin{array}{l}\text { Adultos } \\
(\mathrm{n}=262)\end{array}$} & \multicolumn{2}{|c|}{$\begin{array}{l}\text { Crianças } \\
(\mathrm{n}=84)\end{array}$} \\
\hline & $\mathrm{n}$ & $\%$ & $\mathrm{n}$ & $\%$ \\
\hline Taxa de adesão & \multicolumn{4}{|c|}{$97,0 \%$} \\
\hline Sexo masculino, & 224 & 85,5 & 46 & 54,8 \\
\hline Idade, média \pm dp & \multicolumn{2}{|c|}{$32,2 \pm 7,3$} & \multicolumn{2}{|c|}{$5,8 \pm 3,7$} \\
\hline Estado civil "casado/a" & 125 & 48,1 & - & - \\
\hline \multicolumn{5}{|l|}{ Nível educacional } \\
\hline Ensino básico ou menos & 42 & 16,0 & 84 & 100 \\
\hline Ensino secundário & 89 & 34,0 & - & - \\
\hline Ensino superior & 131 & 50,0 & - & - \\
\hline Ocupação "tem uma profissão" & 167 & 63,7 & - & - \\
\hline \multicolumn{5}{|l|}{ Profissão } \\
\hline $\begin{array}{l}\text { 1. Quadros Superiores da Administração Pública, Dirigentes e Quadros } \\
\text { Superiores de Empresas }\end{array}$ & 18 & 10,0 & - & - \\
\hline 2. Especialistas das Profissões Intelectuais e Científicas & 3 & 1,7 & - & - \\
\hline 3. Técnicos/as e Profissionais de Nível Intermédio & 9 & 5,0 & - & - \\
\hline 4. Pessoal Administrativo e Similares & 3 & 1,7 & - & - \\
\hline 5. Pessoal dos Serviços e Vendedores & 141 & 78,3 & - & - \\
\hline 6. Agricultores e Trabalhadores Qualificados da Agricultura e Pescas & 0 & - & - & - \\
\hline 7. Operários/as, Artífices e Trabalhadores/as Similares & 0 & - & - & - \\
\hline $\begin{array}{l}\text { 8. Operadores/as de Instalações e Máquinas e Trabalhadores/as da } \\
\text { Montagem }\end{array}$ & 2 & 1,1 & - & - \\
\hline Tempo de residência em Portugal, anos, mediana (IIQ) & & & & \\
\hline \multicolumn{5}{|l|}{ Naturalidade } \\
\hline Portugal & 0 & - & 30 & 35,7 \\
\hline País de origem & 261 & 100 & 54 & 64,3 \\
\hline \multicolumn{5}{|l|}{ Religião } \\
\hline Não & 0 & - & 0 & - \\
\hline Católica & 0 & - & 0 & - \\
\hline Judaica & 0 & - & 0 & - \\
\hline Muçulmana & 258 & 99,2 & 84 & 100,0 \\
\hline Hinduísta & 2 & 0,8 & 0 & - \\
\hline Outra Não Cristã & 0 & - & 0 & - \\
\hline \multicolumn{5}{|l|}{ Grau de praticante } \\
\hline 1 - Muito pouco & 166 & 64,6 & 77 & 95,1 \\
\hline 2 - Pouco & 0 & - & 0 & - \\
\hline 3 - Nem pouco nem muito & 4 & 1,6 & 0 & - \\
\hline 4 - Muito & 0 & - & 0 & - \\
\hline 5 - Muitíssimo & 87 & 33,9 & 4 & 4,9 \\
\hline
\end{tabular}

dp - desvio padrão. IIQ - intervalo interquartílico 


\begin{tabular}{|c|c|c|c|c|}
\hline & \multicolumn{4}{|c|}{$\begin{array}{l}\text { Imigrantes Indianos } \\
\qquad(n=348)\end{array}$} \\
\hline & \multicolumn{2}{|c|}{$\begin{array}{l}\text { Adultos } \\
(n=217)\end{array}$} & \multicolumn{2}{|c|}{$\begin{array}{l}\text { Crianças } \\
(n=131)\end{array}$} \\
\hline & $\mathrm{n}$ & $\%$ & $\mathrm{n}$ & $\%$ \\
\hline Taxa de adesão & \multicolumn{4}{|c|}{$95,6 \%$} \\
\hline Sexo masculino, & 149 & 68,7 & 94 & 71,8 \\
\hline Idade, média \pm dp & \multicolumn{2}{|c|}{$38,4 \pm 11,6$} & \multicolumn{2}{|c|}{$8,0 \pm 4,3$} \\
\hline Estado civil "casado/a" & 147 & 68,1 & - & - \\
\hline \multicolumn{5}{|l|}{ Nível educacional } \\
\hline Ensino básico ou menos & 98 & 45,4 & 121 & 96,0 \\
\hline Ensino secundário & 76 & 35,2 & 5 & 4,0 \\
\hline Ensino superior & 42 & 19,4 & & - \\
\hline Ocupação "tem uma profissão" & 134 & 61,8 & 1 & 0,8 \\
\hline \multicolumn{5}{|l|}{ Profissão } \\
\hline $\begin{array}{l}\text { 1. Quadros Superiores da Administração Pública, Dirigentes e Quadros } \\
\text { Superiores de Empresas }\end{array}$ & 3 & 1,7 & - & - \\
\hline 2. Especialistas das Profissões Intelectuais e Científicas & 3 & 1,7 & - & - \\
\hline 3. Técnicos/as e Profissionais de Nível Intermédio & 1 & 0,6 & - & - \\
\hline 4. Pessoal Administrativo e Similares & 0 & 0 & - & - \\
\hline 5. Pessoal dos Serviços e Vendedores & 155 & 90,1 & 1 & 0,8 \\
\hline 6. Agricultores e Trabalhadores Qualificados da Agricultura e Pescas & 1 & 0,6 & - & - \\
\hline 7. Operários/as, Artífices e Trabalhadores/as Similares & 9 & 5,2 & - & - \\
\hline $\begin{array}{l}\text { 8. Operadores/as de Instalações e Máquinas e Trabalhadores/as da } \\
\text { Montagem }\end{array}$ & 0 & 0,0 & - & - \\
\hline Tempo de residência em Portugal, anos, mediana (IIQ) & \multicolumn{2}{|c|}{$8(4-18,8)$} & \multicolumn{2}{|c|}{$6(4-8)$} \\
\hline \multicolumn{5}{|l|}{ Naturalidade } \\
\hline Portugal & 7 & 3,2 & 81 & 61,8 \\
\hline País de origem & 183 & 84,3 & 48 & 36,6 \\
\hline \multicolumn{5}{|l|}{ Religião } \\
\hline Não & 0 & - & 0 & - \\
\hline Católica & 5 & 2,3 & 4 & 3,1 \\
\hline Judaica & 0 & - & 0 & - \\
\hline Muçulmana & 37 & 17,1 & 32 & 24,4 \\
\hline Hinduísta & 141 & 65,0 & 78 & 59,5 \\
\hline Outra Não Cristã & 34 & 15,7 & 17 & 13,0 \\
\hline \multicolumn{5}{|l|}{ Grau de praticante } \\
\hline 1 - Muito pouco & 106 & 48,8 & 84 & 80,0 \\
\hline 2 - Pouco & 6 & 2,8 & 2 & 1,9 \\
\hline 3 - Nem pouco nem muito & 32 & 14,7 & 7 & 6,7 \\
\hline 4 - Muito & 26 & 12,0 & 7 & 6,7 \\
\hline 5 - Muitíssimo & 47 & 21,7 & 5 & 4,8 \\
\hline
\end{tabular}

dp - desvio padrão. IIQ - intervalo interquartílico 


\begin{tabular}{|c|c|c|c|c|}
\hline & \multicolumn{4}{|c|}{$\begin{array}{l}\text { Imigrantes Paquistaneses } \\
\qquad(\mathrm{n}=317)\end{array}$} \\
\hline & \multicolumn{2}{|c|}{$\begin{array}{l}\text { Adultos } \\
(\mathrm{n}=227)\end{array}$} & \multicolumn{2}{|c|}{$\begin{array}{l}\text { Crianças } \\
(\mathrm{n}=90)\end{array}$} \\
\hline & $\mathrm{n}$ & $\%$ & $\mathrm{n}$ & $\%$ \\
\hline Taxa de adesão & \multicolumn{4}{|c|}{$97,8 \%$} \\
\hline Sexo masculino, & 180 & 79,6 & 59 & 55,6 \\
\hline Idade, média \pm dp & \multicolumn{2}{|c|}{$35,5 \pm 8,0$} & \multicolumn{2}{|c|}{$6,9 \pm 3,2$} \\
\hline Estado civil "casado/a" & 144 & 63,4 & - & - \\
\hline \multicolumn{5}{|l|}{ Nível educacional } \\
\hline Ensino básico ou menos & 40 & 17,6 & 86 & 98,9 \\
\hline Ensino secundário & 98 & 43,2 & 1 & 1,1 \\
\hline Ensino superior & 89 & 39,2 & - & - \\
\hline Ocupação "tem uma profissão" & 142 & 62,6 & - & - \\
\hline \multicolumn{5}{|l|}{ Profissão } \\
\hline $\begin{array}{l}\text { 1. Quadros Superiores da Administração Pública, Dirigentes e Quadros } \\
\text { Superiores de Empresas }\end{array}$ & 37 & 25,2 & - & - \\
\hline 2. Especialistas das Profissões Intelectuais e Científicas & 0 & - & - & - \\
\hline 3. Técnicos/as e Profissionais de Nível Intermédio & 2 & 1,4 & - & - \\
\hline 4. Pessoal Administrativo e Similares & 1 & 0,7 & - & - \\
\hline 5. Pessoal dos Serviços e Vendedores & 105 & 71,4 & - & - \\
\hline 6. Agricultores e Trabalhadores Qualificados da Agricultura e Pescas & 0 & - & - & - \\
\hline 7. Operários/as, Artífices e Trabalhadores/as Similares & 0 & - & - & - \\
\hline $\begin{array}{l}\text { 8. Operadores/as de Instalações e Máquinas e Trabalhadores/as da } \\
\text { Montagem }\end{array}$ & 1 & 0,7 & - & - \\
\hline Tempo de residência em Portugal, anos, mediana (IIQ) & \multicolumn{2}{|c|}{$4(2-6)$} & \multicolumn{2}{|c|}{$4(3-4)$} \\
\hline \multicolumn{5}{|l|}{ Naturalidade } \\
\hline Portugal & 0 & - & 18 & 20,0 \\
\hline País de origem & 227 & 100,0 & 72 & 80,0 \\
\hline \multicolumn{5}{|l|}{ Religião } \\
\hline Não & 1 & 0,4 & 0 & - \\
\hline Católica & 0 & - & 0 & - \\
\hline Judaica & 1 & 0,4 & 0 & - \\
\hline Muçulmana & 218 & 96,0 & 90 & 100,0 \\
\hline Hinduísta & 7 & 3,1 & 0 & - \\
\hline Outra Não Cristã & 0 & - & 0 & - \\
\hline \multicolumn{5}{|l|}{ Grau de praticante } \\
\hline 1 - Muito pouco & 111 & 49,3 & 78 & 89,7 \\
\hline 2 - Pouco & 1 & 0,4 & 1 & 1,1 \\
\hline 3 - Nem pouco nem muito & 8 & 3,6 & 0 & - \\
\hline 4 - Muito & 7 & 3,1 & 3 & 3,4 \\
\hline 5 - Muitíssimo & 98 & 43,6 & 5 & 5,7 \\
\hline
\end{tabular}


Tabela 3 - Autoavaliação da qualidade de vida, saúde mental, estado de saúde, estilos de vida e atitudes perante a morte e processo de morte dos adultos da amostra por país de origem

\begin{tabular}{|c|c|c|c|c|c|c|}
\hline & \multicolumn{2}{|c|}{$\begin{array}{l}\text { Imigrantes Bangladeshianos } \\
\text { Adultos }(n=262)\end{array}$} & \multicolumn{2}{|c|}{$\begin{array}{l}\text { Imigrantes Indianos } \\
\text { Adultos }(n=217)\end{array}$} & \multicolumn{2}{|c|}{$\begin{array}{l}\text { Imigrantes Paquistaneses } \\
\text { Adultos }(n=227)\end{array}$} \\
\hline & $\mathrm{n}$ & $\%$ & $\mathrm{n}$ & $\%$ & $\mathrm{n}$ & $\%$ \\
\hline \multicolumn{7}{|c|}{ Indicadores de qualidade de vida } \\
\hline \multicolumn{7}{|l|}{ Qualidade de vida } \\
\hline 1 - Muito má & 1 & 0,4 & 4 & 1,9 & 1 & 0,4 \\
\hline 2 - Má & 1 & 0,4 & 10 & 4,6 & 0 & - \\
\hline 3 - Nem boa nem má & 7 & 2,7 & 51 & 23,6 & 10 & 4,4 \\
\hline 4 - Boa & 184 & 70,8 & 113 & 52,3 & 150 & 66,4 \\
\hline 5 - Muito boa & 67 & 25,8 & 38 & 17,6 & 65 & 28,8 \\
\hline $\begin{array}{l}\text { MHI, "sem possível } \\
\text { sofrimento psicológico" }\end{array}$ & 139 & 53,5 & 132 & 61,1 & 159 & 70,4 \\
\hline \multicolumn{7}{|c|}{ Fatores e comportamentos de risco } \\
\hline \multicolumn{7}{|l|}{ IMC } \\
\hline Baixo peso & 0 & - & 4 & 2,0 & 1 & 0,5 \\
\hline Peso normal & 119 & 47,2 & 86 & 42,6 & 55 & 25,1 \\
\hline Excesso de peso & 128 & 50,8 & 99 & 49,0 & 153 & 69,9 \\
\hline Obesidade & 5 & 2,0 & 13 & 6,4 & 10 & 4,6 \\
\hline Diabetes & 10 & 3,8 & 10 & 4,6 & 4 & 1,8 \\
\hline Dislipidémia & 15 & 5,7 & 25 & 11,5 & 13 & 5,7 \\
\hline Hipertensão arterial & 8 & 3,1 & 20 & 9,2 & 5 & 2,2 \\
\hline Insuficiência cardíaca & 0 & - & 3 & 1,4 & 2 & 0,9 \\
\hline AVC & 2 & 0,8 & 3 & 1,4 & 1 & 0,5 \\
\hline \multicolumn{7}{|l|}{ Hábitos tabágicos } \\
\hline Fumador/a & 49 & 18,8 & 54 & 24,9 & 90 & 40,0 \\
\hline Ex-fumador/a & 3 & 1,1 & 11 & 5,1 & 1 & 0,4 \\
\hline Nunca fumou & 206 & 78,9 & 152 & 70,0 & 134 & 59,6 \\
\hline \multicolumn{7}{|l|}{ Atitudes perante a morte } \\
\hline DAS, média \pm dp & \multicolumn{2}{|c|}{$39,4 \pm 4,4$} & \multicolumn{2}{|c|}{$40,1 \pm 8,5$} & \multicolumn{2}{|c|}{$38,6 \pm 5,1$} \\
\hline $\begin{array}{l}\text { Autorizaria doação de } \\
\text { órgãos }\end{array}$ & 25 & 9,6 & 90 & 42,1 & 35 & 15,8 \\
\hline Autorizaria eutanásia & 25 & 9,6 & 76 & 35,2 & 28 & 12,7 \\
\hline $\begin{array}{l}\text { Autorizaria utilização } \\
\text { prolongada de meios de } \\
\text { suporte de vida }\end{array}$ & 52 & 19,9 & 102 & 47,0 & 56 & 25,6 \\
\hline \multicolumn{7}{|c|}{ Em que país preferia morrer } \\
\hline Portugal & 9 & 3,4 & 46 & 21,3 & 14 & 6,4 \\
\hline País de origem & 237 & 90,8 & 122 & 56,5 & 200 & 91,7 \\
\hline Outro & 1 & 0,4 & 5 & 2,3 & 1 & 0,5 \\
\hline Indiferente & 3 & 1,1 & 27 & 12,5 & 3 & 1,4 \\
\hline
\end{tabular}


Tabela 4 - Saúde oral e utilização dos serviços de saúde nos adultos e crianças da amostra por país de origem

\begin{tabular}{|c|c|c|c|c|c|c|c|c|c|c|c|c|}
\hline & \multicolumn{4}{|c|}{$\begin{array}{l}\text { Imigrantes Bangladeshianos } \\
\qquad(\mathrm{n}=346)\end{array}$} & \multicolumn{4}{|c|}{$\begin{array}{l}\text { Imigrantes Indianos } \\
\qquad(\mathrm{n}=348)\end{array}$} & \multicolumn{4}{|c|}{$\begin{array}{l}\text { Imigrantes Paquistaneses } \\
\qquad(\mathrm{n}=317)\end{array}$} \\
\hline & \multicolumn{2}{|c|}{$\begin{array}{l}\text { Adultos } \\
(\mathrm{n}=262)\end{array}$} & \multicolumn{2}{|c|}{$\begin{array}{l}\text { Crianças } \\
(n=84)\end{array}$} & \multicolumn{2}{|c|}{$\begin{array}{l}\text { Adultos } \\
(\mathrm{n}=217)\end{array}$} & \multicolumn{2}{|c|}{$\begin{array}{l}\text { Crianças } \\
(\mathrm{n}=131)\end{array}$} & \multicolumn{2}{|c|}{$\begin{array}{l}\text { Adultos } \\
(\mathrm{n}=227)\end{array}$} & \multicolumn{2}{|c|}{$\begin{array}{l}\text { Crianças } \\
(n=90)\end{array}$} \\
\hline & $\mathrm{n}$ & $\%$ & $n$ & $\%$ & $\mathrm{n}$ & $\%$ & $\mathrm{n}$ & $\%$ & $\mathrm{n}$ & $\%$ & $\mathrm{n}$ & $\%$ \\
\hline \multicolumn{13}{|l|}{ Saúde oral } \\
\hline \multicolumn{13}{|l|}{$\begin{array}{l}\text { Consultou estomatologista, dentista, higienista ou } \\
\text { outro técnico de saúde dentária }\end{array}$} \\
\hline Sim, há menos de 1 ano & 36 & 13,7 & 11 & 15,9 & 33 & 15,2 & 38 & 29,0 & 43 & 18,9 & 37 & 43,0 \\
\hline Sim, há mais de 1 ano & 119 & 45,4 & 31 & 44,9 & 135 & 62,2 & 73 & 55,7 & 173 & 76,2 & 47 & 54,7 \\
\hline Nunca & 107 & 40,8 & 27 & 39,1 & 49 & 22,6 & 18 & 13,7 & 11 & 4,8 & 2 & 2,3 \\
\hline \multicolumn{13}{|l|}{ Razão para última consulta } \\
\hline Estava com dores ou urgência & 13 & 37,1 & 3 & 27,3 & 7 & 23,3 & 6 & 15,8 & 3 & 7,3 & 7 & 18,9 \\
\hline Extrair um dente & 7 & 20,0 & 2 & 18,2 & 8 & 26,7 & 0 & - & 4 & 9,8 & 2 & 5,4 \\
\hline Prótese dentária (“placa”) & 1 & 2,9 & 0 & - & 3 & 10,0 & 3 & 7,9 & 2 & 4,9 & 4 & 10,8 \\
\hline Conhecer o estado de saúde da boca & 12 & 34,3 & 2 & 18,2 & 2 & 6,7 & 9 & 23,7 & 18 & 43,9 & 13 & 35,1 \\
\hline Visita anual ao dentista & 0 & & 4 & 36,4 & 3 & 10,0 & 15 & 39,5 & 13 & 31,7 & 11 & 29,7 \\
\hline Higienização da boca & 2 & 5,7 & 0 & - & 6 & 20,0 & 3 & 7,9 & 1 & 2,4 & 0 & - \\
\hline Outras razões & 0 & & 0 & - & 0 & - & 2 & 5,3 & 0 & - & 0 & - \\
\hline \multicolumn{13}{|l|}{ Razão para não consultar o dentista } \\
\hline Não precisou & 90 & 84,1 & 25 & 92,6 & 43 & 93,5 & 17 & 100,0 & 10 & 100,0 & 2 & 100,0 \\
\hline É muito caro & 17 & 15,9 & 2 & 7,4 & 3 & 6,5 & 0 & - & 0 & - & 0 & - \\
\hline Consultou em Portugal & 150 & 98,7 & 42 & 100,0 & 145 & 86,8 & 109 & 99,1 & 210 & 97,7 & 84 & 100,0 \\
\hline \multicolumn{13}{|l|}{ Dentição } \\
\hline Tem todos os dentes naturais & 255 & 99,2 & 9 & 13,0 & 172 & 79,6 & 49 & 38,6 & 220 & 96,9 & 10 & 11,8 \\
\hline Faltam alguns dentes naturais & 2 & 0,8 & 59 & 85,5 & 44 & 20,4 & 77 & 60,6 & 7 & 3,1 & 75 & 88,2 \\
\hline Faltam todos os dentes naturais & 0 & & 1 & 1,4 & 0 & - & 0 & - & 0 & - & 0 & - \\
\hline \multicolumn{13}{|l|}{ Prótese } \\
\hline Usa prótese para substituir todos os dentes que faltam & 1 & 100,0 & - & - & 14 & 32,6 & - & & 3 & 60,0 & - & - \\
\hline Não usa prótese & 0 & & - & - & 29 & 67,4 & - & & 2 & 40,0 & - & - \\
\hline \multicolumn{13}{|l|}{ Escova os dentes } \\
\hline Não tem dentes & 0 & & 1 & 1,4 & 0 & - & 2 & 1,7 & 0 & - & 0 & - \\
\hline Nunca & 1 & 0,4 & 2 & 2,9 & 0 & - & 0 & - & 0 & - & 0 & - \\
\hline Às vezes / Menos de 1 vez por dia & 0 & & 1 & 1,4 & 3 & 1,4 & 0 & - & 1 & 0,4 & 1 & 1,2 \\
\hline Uma vez ao dia & 32 & 12,4 & 39 & 56,5 & 44 & 20,4 & 51 & 42,9 & 15 & 6,6 & 45 & 53,6 \\
\hline Duas ou mais vezes ao dia & 225 & 87,2 & 26 & 37,7 & 169 & 78,2 & 65 & 54,6 & 211 & 93,0 & 38 & 45,2 \\
\hline \multicolumn{13}{|l|}{ Utilização dos serviços de saúde } \\
\hline \multicolumn{13}{|l|}{$\begin{array}{l}\text { Dificuldade linguística na compreensão nos serviços } \\
\text { de saúde }\end{array}$} \\
\hline Nunca recorreu aos serviços de saúde & 4 & 1,5 & 0 & - & 7 & 3,2 & 2 & 1,6 & 1 & 0,4 & 1 & 1,1 \\
\hline Não & 218 & 84,2 & 79 & 94,0 & 151 & 69,9 & 115 & 89,1 & 194 & 85,5 & 84 & 93,6 \\
\hline Sim & 37 & 14,3 & 5 & 6,0 & 58 & 26,9 & 11 & 8,5 & 32 & 14,1 & 5 & 5,6 \\
\hline $\begin{array}{l}\text { Dificuldade fez com que não recorresse aos serviços } \\
\text { de saúde }\end{array}$ & 2 & 5,4 & 0 & - & 5 & 8,9 & 0 & - & 3 & 8,6 & 0 & - \\
\hline Foi recusada a prestação de cuidados de saúde & 3 & 1,2 & 0 & - & 15 & 7,2 & 5 & 4,0 & 18 & 8,0 & 2 & 2,2 \\
\hline $\begin{array}{l}\text { Foi tratado/a de forma discriminatória por ser } \\
\text { imigrante pelos/as profissionais de saúde }\end{array}$ & 0 & - & 0 & - & 4 & 1,9 & 0 & - & 2 & 0,9 & 0 & - \\
\hline
\end{tabular}

\begin{abstract}
De uma maneira geral, considera que os/as profissionais de saúde compreendem bem as
\end{abstract} necessidades de saúde dos/as imigrantes?

\title{
Concordo totalmente
}

Concordo parcialmente

Não concordo nem discordo

Discordo parcialmente

Discordo totalmente

Inscrição num Centro de Saúde

Tem médico/a de família

Recorreu ao serviço de urgência

Desde que está em Portugal, já recorreu a cuidados de saúde fora de Portugal?

Nos últimos 12 meses, houve alguma vez que tenha precisado de comprar ou pagar algum dos seguintes tratamentos e não o fez por não poder comportar os custos

\begin{tabular}{|c|c|c|c|c|c|c|c|c|c|c|c|c|}
\hline Medicamentos & 3 & 1,1 & 0 & - & 16 & 7,4 & 4 & 3,1 & 5 & 2,2 & 0 & - \\
\hline Médicos/as & 3 & 1,1 & 0 & - & 9 & 4,2 & 2 & 1,5 & 4 & 1,8 & 0 & - \\
\hline Meios complementares de diagnóstico & 2 & 0,8 & 0 & - & 8 & 3,7 & 0 & - & 4 & 1,8 & 0 & - \\
\hline
\end{tabular}


indivíduos com excesso de peso e obesidade e de fumadores. Apesar disto, a comunidade Indiana é quem tem uma maior proporção de doenças crónicas.

\section{DISCUSSÃO E RECOMENDAÇÕES}

Pretendia-se com este estudo obter informação que servisse de base para a elaboração de recomendações para promover boas práticas na prestação de serviços e gestão de recursos do SNS, direcionadas para a população imigrante do subcontinente indiano em Portugal. Estas recomendações são adaptadas a partir das recomendações da OMS utilizando dados recolhidos por questionários junto desta população.

Quando comparada com os imigrantes inquiridos no INS 2005/2006 (INS), ${ }^{6}$ a amostra deste estudo tinha uma maior proporção de indivíduos do sexo masculino $(74 \%$ e $51 \%$ ) e de inquiridos a viver em Portugal há cinco anos ou menos (67\% neste estudo e $28 \%$ no INS). Quando comparada com os dados censitários de 2011 referentes à população residente em Portugal de nacionalidade Bangladeshiana, Indiana e Paquistanesa, esta amostra é semelhante em algumas características: a maioria são homens, jovens, muçulmanos (Paquistaneses e Bangladeshianos) ou hinduístas (Indianos). Por outro lado, a amostra tem uma proporção de indivíduos com ensino superior consideravelmente maior do que os dados censitários. ${ }^{3}$ Assim, apesar da informação censitária se referir apenas a indivíduos naturais destes países, enquanto que esta amostra inclui imigrantes de segunda geração, os dados apontam para uma sobreposição das características da amostra com as da população de onde esta é retirada.

De uma forma geral, os imigrantes do subcontinente indiano mostraram melhores indicadores de saúde quando comparados com a amostra de imigrantes do INS: mais adultos classificaram a sua qualidade de vida como 'boa' ou 'muito boa' ( $87 \%$ vs $61 \%$ no INS) e menos referiram ter hipertensão arterial ( $5 \%$ vs 13\%). No entanto, mais adultos do subcontinente indiano foram classificados como tendo provável sofrimento psicológico ( $39 \%$ vs $22 \%$ ) e uma proporção ligeiramente maior referiu ter hábitos tabágicos atuais ( $27 \%$ vs $22 \%)$.

Sendo em geral mais novos, não é surpreendente que esta amostra tenha melhores indicadores de saúde. No entanto, a maior frequência de provável sofrimento psicológico pode ser um alerta para necessidades específicas de cuidados de saúde desta subpopulação.

Apenas entre 9,6 e 42,1\% dos inquiridos do subcontinente indiano autorizaria a doação de órgãos após a morte, contrastando com $85,7 \%$ da população portuguesa. ${ }^{28}$ Este resultado alerta para a importância de ter em conta as diferenças culturais e de informar as populações imigrantes adequadamente.

Dias e colaboradores ${ }^{8}$ procuraram caracterizar o acesso aos cuidados de saúde da população imigrante, colhendo informação junto de cerca de 1500 imigrantes no Centro Nacional de Apoio ao Imigrante em Lisboa. Cerca de metade desta amostra tinha nacionalidade sul-americana, seguida de africana (35\%), europeia de leste (12\%) e asiática (3\%). Cerca de metade era do sexo masculino (53\%) e tinham, em média, $33 \pm 9$ anos de idade (a amostra de imigrantes do subcontinente indiano tinha, em média, $35 \pm$ 9 anos de idade). Nesta amostra, $79 \%$ já tinham utilizado o SNS em Portugal, dos quais apenas $1 \%$ identificaram dificuldades linguísticas na utilização dos serviços de saúde. Em comparação, 98\% dos imigrantes do subcontinente indiano já tinham utilizado o SNS, dos quais $18 \%$ identificaram a língua como uma barreira à utilização dos cuidados de saúde.

Para investigar os fatores associados à utilização do SNS, Dias e colaboradores ${ }^{9}$ utilizaram uma técnica de bola de neve para obter uma amostra de cerca de 1300 imigrantes do Brasil, países Africanos de língua oficial portuguesa e países da Europa de Leste a viver na região de Lisboa. Nesta amostra, cerca de um terço tinha ensino superior completo (31\%), cerca de metade eram do sexo masculino (49\%) e a média de idades foi de $36 \pm 12$ anos. Aproximadamente $77 \%$ dos inquiridos já tinham utilizado o SNS em Portugal, uma proporção mais baixa do que a encontrada junto dos imigrantes do subcontinente indiano (98\%).

A população do subcontinente indiano teve, quando comparada com populações imigrantes de outras nacionalidades em Lisboa, melhores indicadores de saúde, maior utilização do SNS e reportou dificuldades linguísticas nesta utilização mais frequentemente. A interpretação do número de inscritos no centro de saúde deve ser feita cautelosamente, já que pode ser motivada pelo processo de legalização no País. ${ }^{29}$

A técnica de amostragem utilizada pode ter implicações importantes na interpretação dos resultados, uma vez que poderá ter contribuído para uma considerável homogeneidade da amostra. No entanto, esta técnica é a mais adequada para populações relativamente pequenas e de difícil acesso, já que permite obter um considerável número de participantes que de outra forma seria difícil e permite incluir os imigrantes em situação regular e irregular.

A interpretação dos resultados deste estudo é ainda limitada pela falta de estudos que permitam uma comparação adequada. Os estudos existentes ou não se destinam diretamente à população imigrante, o que limita a sua representatividade (como é o caso do INS), ou têm como objetivo principal a caracterização da utilização dos cuidados de saúde (e não da saúde) desta população. Particularmente, no que diz respeito ao INS, é de ter em conta que a amostra de imigrantes inclui pessoas de diferentes nacionalidades daquelas representadas neste estudo e não inclui indivíduos em situação irregular.

Não obstante destas limitações, este estudo alcançou uma taxa de adesão elevada junto de uma população de difícil acesso, obtendo assim informação acessível com pouca frequência. Com base nesta informação, é possível adaptar as recomendações existentes, produzindo as seguintes dez recomendações.

1. Formar os profissionais de saúde em competências para interagir com utentes imigrantes do 
Tabela 5 - Características sociodemográficas e de saúde, ajustadas para a idade, por país de origem

\begin{tabular}{|c|c|c|c|}
\hline Variáveis & Bangladesh & Índia & Paquistão \\
\hline Idade (média \pm dp) & $\begin{array}{c}32,2 \pm 7,3 \\
(31,3 ; 33,0)\end{array}$ & $\begin{array}{l}38,4 \pm 11,6 \\
(36,9 ; 39,9)\end{array}$ & $\begin{array}{c}35,5 \pm 8,0 \\
(34,4 ; 36,5)\end{array}$ \\
\hline Sexo feminino $(\%)$ & $\begin{array}{c}13,9 \\
(10,2 ; 17,8)\end{array}$ & $\begin{array}{c}30,5 \\
(24,4 ; 36,5)\end{array}$ & $\begin{array}{c}19,8 \\
(14,2 ; 25,3)\end{array}$ \\
\hline Casado (\%) & $\begin{array}{c}52,3 \\
(46,7 ; 57,9)\end{array}$ & $\begin{array}{c}64,5 \\
(59,5 ; 69,5)\end{array}$ & $\begin{array}{c}61,5 \\
(56,4 ; 66,6)\end{array}$ \\
\hline Com ensino superior $(\%)$ & $\begin{array}{c}49,1 \\
(43,0 ; 55,2)\end{array}$ & $\begin{array}{c}19,8 \\
(14,4 ; 25,1)\end{array}$ & $\begin{array}{c}34,9 \\
(29,1 ; 40,8)\end{array}$ \\
\hline Desempregado (\%) & $\begin{array}{c}14,1 \\
(10,0 ; 18,2)\end{array}$ & $\begin{array}{c}5,0 \\
(2,1 ; 7,9)\end{array}$ & $\begin{array}{c}0,4 \\
(0,0 ; 1,1)\end{array}$ \\
\hline Tempo de residência em Portugal, anos (mediana) & $\begin{array}{c}1 \\
(1-4)\end{array}$ & $\begin{array}{c}8 \\
(4-18,8)\end{array}$ & $\begin{array}{c}4 \\
(2-6)\end{array}$ \\
\hline Natural de Portugal $(\%)^{*}$ & $\begin{array}{c}3,1 \\
(2,1 ; 3,9)\end{array}$ & $\begin{array}{c}10,8 \\
(4,2 ; 17,4)\end{array}$ & $\begin{array}{c}2,3 \\
(1,4 ; 3,2)\end{array}$ \\
\hline Religião predominante * & Muçulmana & Hindu & Muçulmana \\
\hline Autoavaliação da qualidade de vida como boa ou muito boa (\%) & $\begin{array}{c}96,4 \\
(94,0 ; 98,7)\end{array}$ & $\begin{array}{c}70,2 \\
(64,6 ; 75,8)\end{array}$ & $\begin{array}{c}95,2 \\
(92,4 ; 97,9)\end{array}$ \\
\hline Possível sofrimento psicológico (\%) & $\begin{array}{c}46,1 \\
(40,1 ; 52,1)\end{array}$ & $\begin{array}{c}38,2 \\
(31,8 ; 44,7)\end{array}$ & $\begin{array}{c}29,1 \\
(22,7 ; 35,6)\end{array}$ \\
\hline Excesso de peso e obesidade (\%) & $\begin{array}{c}54,1 \\
(47,9 ; 60,3)\end{array}$ & $\begin{array}{c}55,4 \\
(47,1 ; 60,3)\end{array}$ & $\begin{array}{c}74,7 \\
(69,4 ; 80,1)\end{array}$ \\
\hline Dificuldade linguística na compreensão nos serviços de saúde (\%) & $\begin{array}{c}14,7 \\
(10,3 ; 18,9)\end{array}$ & $\begin{array}{c}26,7 \\
(22,1 ; 31,1)\end{array}$ & $\begin{array}{c}13,4 \\
(9,1 ; 17,7)\end{array}$ \\
\hline Dificuldade fez com que não recorresse aos serviços de saúde (\%) & $\underset{* *}{5,2}$ & $\begin{array}{c}7,9 \\
(1,4 ; 14,3)\end{array}$ & $\begin{array}{c}7,9 \\
* *\end{array}$ \\
\hline Foi recusada a prestação de cuidados de saúde (\%) & $\begin{array}{c}1,1 \\
(0,6 ; 3,0)\end{array}$ & $\begin{array}{c}6,7 \\
(3,5 ; 9,9)\end{array}$ & $\begin{array}{c}7,4 \\
(4,1 ; 10,7)\end{array}$ \\
\hline $\begin{array}{l}\text { Foi tratado de forma discriminatória por ser imigrante pelos profissionais de } \\
\text { saúde }(\%)\end{array}$ & 0,0 & $\begin{array}{c}1,9 \\
(0,0 ; 3,8)\end{array}$ & $\begin{array}{c}0,7 \\
(0,0 ; 1,7)\end{array}$ \\
\hline $\begin{array}{l}\text { Concorda que, de uma maneira geral, os profissionais de saúde compreendem } \\
\text { bem as necessidades de saúde dos imigrantes (\%) }\end{array}$ & $\begin{array}{c}97,9 \\
(96,2 ; 99,7)\end{array}$ & $\begin{array}{c}90,9 \\
(86,8 ; 94,9)\end{array}$ & $\begin{array}{c}98,4 \\
(96,8 ; 99,9)\end{array}$ \\
\hline Inscrição num Centro de Saúde (\%) & $\begin{array}{c}97,1 \\
(95,0 ; 99,2)\end{array}$ & $\begin{array}{c}91,8 \\
(88,0 ; 95,5)\end{array}$ & $\begin{array}{c}99,6 \\
(98,9 ; 100,0)\end{array}$ \\
\hline Tem médico de família (\%) & $\begin{array}{c}95,9 \\
(93,5 ; 98,3)\end{array}$ & $\begin{array}{c}85,6 \\
(80,8 ; 90,4)\end{array}$ & $\begin{array}{c}96,5 \\
(94,1 ; 98,9)\end{array}$ \\
\hline Recorreu ao serviço de urgência (\%) & $\begin{array}{c}17,9 \\
(13,1 ; 22,7)\end{array}$ & $\begin{array}{c}40,7 \\
(34,3 ; 47,0)\end{array}$ & $\begin{array}{c}18,1 \\
(12,5 ; 23,6)\end{array}$ \\
\hline $\begin{array}{l}\text { Não comprou medicamentos nos últimos } 12 \text { meses por não poder comportar } \\
\text { os custos }(\%)\end{array}$ & $\begin{array}{c}1,2 \\
(0,0 ; 2,5)\end{array}$ & $\begin{array}{c}7,4 \\
(3,9 ; 10,9)\end{array}$ & $\begin{array}{c}2,1 \\
(0,3 ; 4,0)\end{array}$ \\
\hline Não foi ao médico nos últimos 12 meses por não poder comportar os custos (\%) & $\begin{array}{c}1,2 \\
(0,0 ; 2,5)\end{array}$ & $\begin{array}{c}4,2 \\
(1,9 ; 6,5)\end{array}$ & $\begin{array}{c}1,6 \\
(0,0 ; 3,2)\end{array}$ \\
\hline $\begin{array}{l}\text { Não fez algum meio complementar de diagnóstico nos últimos } 12 \text { meses por } \\
\text { não poder comportar os custos (\%) }\end{array}$ & $\begin{array}{c}0,8 \\
(0,0 ; 1,8)\end{array}$ & $\begin{array}{c}3,6 \\
(1,2 ; 5,9)\end{array}$ & $\begin{array}{c}1,8 \\
(0,0 ; 2,9)\end{array}$ \\
\hline Nunca consultou um técnico de saúde dentária (\%) & $\begin{array}{c}39,6 \\
(33,8 ; 45,4)\end{array}$ & $\begin{array}{c}14,8 \\
(10,2 ; 19,4)\end{array}$ & $\begin{array}{c}4,9 \\
(2,1 ; 7,7)\end{array}$ \\
\hline
\end{tabular}

dp: desvio-padrão; * amostra de adultos e crianças (todos os outros referem-se apenas a adultos); ** não foi possível calcular o intervalo de confiança.

Notas: intervalo de confiança de $95 \%$ ou intervalo interquartílico entre parêntesis.

subcontinente indiano apenas quando os profissionais manifestem esta necessidade.

Um estudo em Portugal identificou oportunidades de intervenção nas capacidades socioculturais dos profissionais de saúde, ${ }^{11}$ mas $94 \%$ dos imigrantes deste estudo consideram que os profissionais de saúde compreendem bem as necessidades de saúde dos imigrantes do subcontinente indiano, não parecendo assim que exista necessidade de formação nesse sentido.

2. Formar os profissionais de saúde sobre as disposições legais que regem o acesso aos cuidados de saúde pelos indivíduos imigrantes. 
Foram documentados casos de unidades de saúde em Portugal que recusaram indevidamente o acesso a imigrantes que tinham direito a cuidados de saúde. ${ }^{10}$ Entre a amostra deste estudo, já foi recusada a prestação de cuidados de saúde a $5 \%$ dos inquiridos, o que reforça esta necessidade.

3. Reforçar a disponibilidade dos serviços de interpretação para os imigrantes do subcontinente indiano que recorrem aos cuidados de saúde e que não sejam fluentes na língua portuguesa.

As barreiras de língua são das mais frequentemente identificadas no que diz respeito ao acesso aos cuidados de saúde por parte de populações imigrantes. Muitos imigrantes deste subcontinente não são fluentes na língua portuguesa (neste estudo, todos os questionários foram respondidos em inglês) e cerca de $18 \%$ refere já ter tido dificuldade linguística na compreensão quando tentou aceder aos serviços de saúde em Portugal.

4. Fornecer informação aos profissionais de saúde sobre os problemas de saúde mais prevalentes e problemas particulares da população imigrante do subcontinente indiano.

A informação sobre as características epidemiológicas de uma população dará aos profissionais de saúde um conhecimento acrescido sobre esta população, tal como recomenda a $\mathrm{OMS}^{25}$, permitindo uma melhoria na qualidade dos cuidados fornecidos.

5. Monitorizar a saúde e a utilização dos cuidados de saúde pela população imigrante do subcontinente indiano.

Tal como recomendado pela OMS, um sistema de monitorização poderá informar acerca das necessidades de saúde e da acessibilidade deste grupo aos cuidados de saúde. Esta recomendação é particularmente importante face a ausência de conhecimento sobre o estado de saúde e a utilização dos cuidados de saúde por parte desta população.

6. Considerar a criação de trabalhadores comunitários de saúde específicos para cada população, de forma a implementar ações preventivas e informar sobre o acesso aos cuidados de saúde.

As três comunidades estudadas apresentaram perfis bastante diferentes em termos de saúde e de utilização dos cuidados de saúde. Apesar de se mostrarem, em geral, satisfeitas com os profissionais de saúde em Portugal, a vulnerabilidade das populações imigrantes e a identificação de várias barreiras à utilização dos cuidados de saúde salientam a necessidade de intervenções dirigidas. A criação da figura de 'trabalhador comunitário de saúde' pode ajudar na melhoria do estado de saúde desta população e promover a utilização adequada dos serviços de saúde. ${ }^{25}$

7. Consultar representantes da comunidade imigrante do subcontinente indiano de forma a conhecer as necessidades específicas deste grupo populacional.

Para além da evidência existente sobre os problemas particulares desta comunidade, é possível e provável que nem todos os seus problemas particulares se reflitam na investigação feita, pelo que é importante ouvir diretamente a população-alvo das intervenções, tal como recomenda a OMS. $^{25}$

8. Informar os imigrantes do subcontinente indiano, possivelmente através dos seus médicos de família, sobre a legislação que regula a doação de órgãos em Portugal.

Em Portugal é necessário deixar por escrito a vontade individual de não doar órgãos após a morte. ${ }^{30}$ Este estudo mostrou que muitos imigrantes do subcontinente indiano, particularmente do Bangladesh, não concordam com a doação de órgãos, contrastando com a opinião da população portuguesa. ${ }^{28}$ É necessário, assim, garantir que a vontade destes indivíduos é respeitada e informá-los dos seus direitos.

\section{CONCLUSÃO}

O conhecimento sobre a saúde e o acesso aos cuidados de saúde dos imigrantes do subcontinente indiano em Portugal é escasso. Este estudo adicionou conhecimento previamente inexistente e permitiu o desenvolvimento de recomendações adaptadas à promoção da saúde desta população imigrante nos cuidados de saúde em Portugal.

Este estudo permitiu também identificar lacunas no conhecimento que implicarão um esforço por parte de investigadores, profissionais de saúde e policy-makers para preencher. Entre estas incluem-se a aparente discrepância entre a autoperceção dos profissionais de saúde em Portugal e a perceção dos imigrantes do subcontinente indiano sobre as capacidades socioculturais destes profissionais. A conjugação das recomendações com os dados populacionais também beneficiaria de uma integração por um conjunto de peritos no assunto, possivelmente utilizando um método Delphi de forma a reunir opiniões e completar as recomendações.

Por fim, esta investigação demonstra a importância de tomar decisões referentes aos serviços de saúde informadas pela evidência e que revele a importância de um sistema integrado de monitorização não só para fins de investigação mas acima de tudo para melhorar os serviços de saúde e a saúde dos grupos populacionais mais vulneráveis.

\section{AGRADECIMENTOS}

Os autores gostariam de agradecer a todos os participantes pela colaboração no estudo, ao Centro Nacional de Apoio ao Imigrante de Lisboa, à Associação Solidariedade Imigrante - Associação para a Defesa dos Direitos dos Imigrantes, e à Comunidade Hindu de Portugal pela facilitação das instalações para a administração dos questionários. Por fim, gostariam de agradecer à equipa de inquiridores e aos colaboradores do projeto, nomeadamente Filipe Leão Miranda e Rui Simões. 


\section{CONFLITO DE INTERESSES}

Os autores declaram não ter nenhum conflito de interesses.

\section{REFERÊNCIAS}

1. Instituto Nacional de Estatística. A população estrangeira em Portugal. Lisboa: INE; 2012.

2. Serviço de Estrangeiros e Fronteiras. Relatório de imigração, fronteiras e asilo. Lisboa: SEF / Gabinete de Estudos, Planeamento e Formação; 2012.

3. Instituto Nacional de Estatística. Censos 2011 - População residente da China, Brasil, Bangladesh, Cabo-Verde, Guiné-Bissau, Paquistão e Índia, por sexo, segundo o grupo etário, o nível de escolaridade completo, o estado civil, a profissão e a religião. Lisboa: INE; 2013.

4. Machado FL. Migrações, saúde e doença-que investigação em Portugal? Migrações-Imigração Saúde. 2007;1:201-3.

5. Fonseca ML, Silva S, Esteves A, McGarrigle J. MIGHEALTHNETPortuguese State of the Art Report. J Epidemiol. 2009;35:270-6.

6. Dias $\mathrm{CM}$, Paixão E, Branco MJ, Falcão JM. A saúde dos imigrantes. Inquérito Nacional de Saúde 2005-2006. Lisboa: INS; 2008.

7. Gonçalves A, Dias S, Luck M, Fernandes MJ, Cabral J. Acesso aos cuidados de saúde de comunidades migrantes: problemas e perspectivas de intervenção. Rev Port Saúde Pública. 2003;21:55-64

8. Dias SF, Severo M, Barros H. Determinants of health care utilization by immigrants in Portugal. BMC Health Serv Res. 2008;8:207.

9. Dias S, Gama A, Cortes M, de Sousa B. Healthcare-seeking patterns among immigrants in Portugal. Health Soc Care Community. 2011;19:514-21.

10. Fonseca ML, Ormond M, Malheiros J, Patrício M, Martins F. Reunificação familiar e imigração em Portugal: Alto-Comissariado para a Imigração e Minorias Étnicas. Lisboa: Observatório da Imigração; 2005.

11. Dias S, Gama A, Silva AC, Cargaleiro $H$, Martins MO. Barreiras no acesso e utilização dos serviços de saúde pelos imigrantes: a perspectiva dos profissionais de saúde. Acta Med Port. 2011;24:511-6.

12. Ahmed SM, Lemkau JP. Cultural issues in the primary care of South Asians. J Immigr Health. 2000;2:89-96.

13. Shibusawa T, MuiAC. Health status and health services utilization among older Asian Indian immigrants. J Immigr Minor Health. 2010;12:527-33.

14. Singh M. The utilization of mental health services by south-asian immigrant communities in the United States. Columbia Univ J Stud Soc Work. 2003;1:15-20.

15. Gatrad A. Muslim customs surrounding death, bereavement, postmortem examinations, and organ transplants. BMJ. 1994;309:521-3.

16. Searight HR, Gafford J. Cultural diversity at the end of life: issues and guidelines for family physicians. Am Fam Physician. 2005;71:515-22.

17. Ávila P, Alves M. Da Índia a Portugal-trajectórias sociais e estratégias colectivas dos comerciantes indianos. Sociol: Probl Prát. 1993;115-33.

\section{FONTES DE FINANCIAMENTO}

O estudo SAIMI foi financiado pela Direção-Geral da Saúde (DGS).

18. Dias N. Beyond familial dharma-hindu entrepreneurial behaviour in a migratory context. Immigration and Place in Mediterranean Metropolises. Lisboa: Luso-American Foundation; 2002.

19. Bastos SP, Bastos JG. Filhos diferentes de deuses diferentes. Manejos da religião em processos de inserção social diferenciada: uma abordagem estrutural dinâmica. Lisboa: ACIDI, IP; 2006

20. Alves M, Ávila P. Indianos em Portugal: processos e (re) construção de identidade e estratégias de integração. AA VV, dinâmicas culturais, cidadania e desenvolvimento local, actas do Encontro em Vila do Conde. Vila do Conde: Associação Portuguesa de Sociologia; 1994.

21. Bastos ST. A comunidade hindu da quinta da holandesa: um estudo antropológico sobre a organização sócio-espacial da casa. Lisboa: LNEC/ITECS; 1990.

22. Bastos SP. Espaço doméstico, espaço simbólico e identidade-Um olhar sobre o viver indiano na cidade de Lisboa. Colóquio viver $(n)$ a cidade. 1990:17-31.

23. Mapril J. «Bangla masdjid»: Islão e bengalidade entre os bangladeshianos em Lisboa. Análise Social. 2005;39:851-73.

24. O'Donnell C, Burns N, Dowrick C, Lionis C, MacFarlane A. Health-care access for migrants in Europe. Lancet. 2013;382:393.

25. Organização Mundial da Saúde. How health systems can address health inequities linked to migration and ethnicity. Copenhaga: WHO Regional Office for Europe; 2010.

26. Governo de Portugal. II Plano para a Integração dos Imigrantes 20102013. In: Presidência do Conselho de Ministros, Alto Comissariado para a Imigração e Diálogo Intercultural IP, Fundo Europeu para a Integração de Nacionais de Países Terceiros, editors. Resolução do Conselho de Ministros no 74/2010.

27. Dunn J, Ferri CP. Epidemiological methods for research with drug misusers: review of methods for studying prevalence and morbidity. Rev Saúde Pública. 1999;33:206-15

28. DECO. Segundo sopro de vida. Proteste Saúde n81 Outubro / Novembro 2009.

29. Assembleia da República Portuguesa. Lei $n^{\circ} 23 / 2007$ de 4 de Julho. Aprova o regime jurídico de entrada, permanência, saída e afastamento de estrangeiros do território nacional. In: Diário da República $1^{\text {a }}$ série No 127 .

30. Governo de Portugal. Objeção à doação de órgãos para transplante [consultado 2014 Mar 5]. Disponível em: http:// www.portaldasaude.pt/portal/conteudos/informacoes+uteis/ doacao+de+orgaos+e+transplantes/objecao_doacao.htm. 
Inês CAMPOS MATOS, Violeta ALARCÃO, Elisa LOPES, Carla OIKO, Mário CARREIRA

\section{Estudo SAIMI - Saúde e Acesso aos Serviços de Saúde dos Imigrantes do Subcontinente Indiano em Lisboa: Que Recomendações para Cuidados de Saúde Equitativos e Culturalmente Adaptados?}

Acta Med Port 2015:28:164-176

Publicado pela Acta Médica Portuguesa, a Revista Científica da Ordem dos Médicos

Av. Almirante Gago Coutinho, 151

1749-084 Lisboa, Portugal.

Tel: +351218428215

E-mail: submissao@actamedicaportuguesa.com

www.actamedicaportuguesa.com

ISSN:0870-399X | e-ISSN: 1646-0758

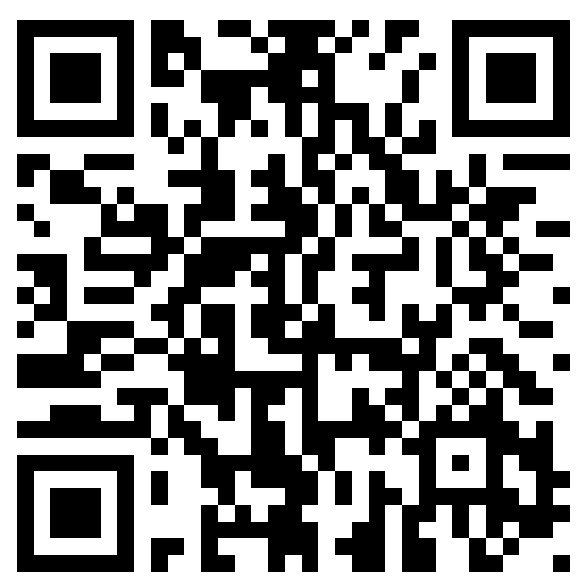

